Results: In all, $4 \cdot 6 \%$ of the children were obese $(4 \cdot 9 \%$ for boys and $4 \cdot 2 \%$ for girls) and $14 \%$ overweight (14.6\% for boys and $13 \cdot 3 \%$ for girls). Weight status re-evaluation demonstrated that overweight rate was decreased for the IG $(-3 \cdot 7 \%)$, while it was increased $(+3 \cdot 5 \%)$ for the CG. However this difference was not statistically significant. Food consumption frequency evaluation at baseline showed that the rate of unhealthy snacking was high in preschool children in northern Greece and it was higher for overweight and obese children. Overweight children consumed more often milk and yoghurt with sugar $(P=0 \cdot 044)$, candies $(P=0 \cdot 00)$ and sugar-sweetened drinks $(P=0 \cdot 003)$ compared with normal weight children. After the nutrition intervention was completed, the re-evaluation showed that the frequency of consumption of unhealthy snacks such as potato chips $(P=0 \cdot 028)$, packaged pastries and confectionery $(P=0.025)$ and bottled sweetened juices $(P=0 \cdot 00)$ decreased in the IG and the decrease was higher for the overweight children, compared with the CG.

Conclusions: The study showed that a nutrition intervention program, even of a short duration, focusing on preschool children can affect food choices and decrease the frequency of unhealthy snack consumption.

\title{
The effects of a community weight loss program for children and young people in nine locations across England
}

\author{
P Gately ${ }^{\top}$ and Y Muhtadi ${ }^{2}$
}

${ }^{1}$ Carnegie Weight Management, Carnegie Faculty of Sport and Education, Leeds Metropolitan University, Leeds, UK: ${ }^{2}$ Human Nutrition Unit, The University of Sheffield, Sheffield, UK

Introduction: Childhood obesity continues to be a public health challenge. Although a range of intervention studies demonstrate it is possible to achieve short-term lifestyle changes and weight loss, many studies are one off trials. Given the scale of the problem, in addition to positive outcomes, replication of the positive outcomes in a range of groups and locations is necessary. The present study reports the outcomes of twenty-eight programmes delivered in nine locations across the England.

Method: Five hundred and eighty-seven participants, aged 4-17 years, were included from twenty-eight Carnegie Club programmes across nine different locations in England. The 12-week programme included parallel child and parent sessions for $3.5 \mathrm{~h}$ each week. All sessions consisted of lifestyle education and physical activity. All participants were assessed weekly for body mass, with pre- and post-measures collected for stature, BMI, BMI SD Score, waist circumference, \% body fat, blood pressure and global self-worth. All delivery staff undertook a comprehensive training programme and used age appropriate standardized materials for programme delivery.

Results: Over the 12-week period, significant $(P<0 \cdot 01)$ reductions were observed in body mass, BMI, BMI SD score, waist circumference, \% body fat and blood pressure. Global self-worth and height increased significantly $(P<0 \cdot 01)$. No differences were observed in any variable between the programmes or locations.

Conclusions: The Carnegie clubs demonstrated significant short-term beneficial outcomes in anthropometry, adiposity, blood pressure and psychological well-being among overweight and obese children and adolescents. The lack of variation between outcomes demonstrates the reliability of this treatment model.

\section{2nd Workshop: Climate Change and Childhood Obesity - Keynote Speaker}

\section{Climate and fat metabolism}

\section{Saverio Cinti}

Professor of Human Anatomy, University of Ancona; President of the Teaching Committee of the Faculty of Medicine and President Italian Society of Obesity (2008/2010) 